Pacific Journal of Mathematics

ON NILPOTENCY AND RESIDUAL FINITENESS IN 


\section{ON NILPOTENCY AND RESIDUAL FINITENESS IN SEMIGROUPS}

\section{Gerard LALLEMENT}

It is proved that the class $\mathscr{C}$ of regular nilpotent semigroups coincides with the class of semilattices of nilpotent groups. Consequently, finitely generated semigroups in the class $\mathscr{C}$ are residually finite. The same results are true for semisimple 2-nilpotent semigroups.

1. Introduction. For semigroups defined in terms of generators and relations, the word problem is known to be recursively unsolvable in general (Post, [15]) but finitely presented semigroups which are residually finite do have a solvable word problem (McKinsey [13], T. Evans [3]). Although considerable work has been done to find large classes of residually finite groups (see e.g. the expository paper of W. Magnus [9]) only a few papers deal with residual finiteness in semigroups. Among the known results and apart from the solvability of the word problem, let us mention that any finitely generated residually finite semigroup is hopfian [4] and has a residually finite semigroup of endomorphisms [5]. Concerning classes of residually finite semigroups, one of the most significant results is due to A. I. Malcev who proved that finitely generated abelian semigroups are residually finite [12] (see also [1]). In trying to extend Malcev's result, one might recall an early result in group theory: Polycyclic, and in particular finitely generated nilpotent groups are residually finite (Hirsch [6]). A. I. Malcev [11], B. H. Neumann and Tekla Taylor [14] have shown that nilpotency of class $c$ could be defined in group theory by the use of a law $L_{c}$ not involving inverses. We shall recall the definition of $L_{c}$ in the next section and adopt it as a definition of nilpotent semigroups. We then ask the following question:

Are finitely generated nilpotent semigroups residually finite? We show, (Corollary 3.1), that the answer is yes for finitely generated nilpotent regular (in the Von Neumann's sense) semigroups. Attempts to remove the regularity restriction in particular cases, (see Corollary 4.2) and examples, (see 4.5) lead us to consider that a positive answer to the question is not unreasonable. I am indebted to R. P. Hunter for drawing my attention to this problem.

2. Nilpotent semigroups. As in [14], we define the variety of nilpotent semigroups of class $c$ inductively as follows: Let $q_{1}, q_{2}, \cdots, q_{c}$ be words in the variables $x, y, z_{1}, z_{2}, \cdots$, such that $q_{1}(x, y)=x y$ and 


$$
q_{i+1}\left(x, y, z_{1}, z_{2}, \cdots z_{i}\right)=q_{i}\left(x, y, z_{1}, \cdots, z_{i-1}\right) z_{i} q_{i}\left(y, x, z_{1}, \cdots, z_{i-1}\right) \text {. }
$$

Let $L_{c}$ be the law

$$
L_{c}: q_{c}\left(x, y, z_{1}, \cdots, z_{c-1}\right)=q_{c}\left(y, x, z_{1}, \cdots, z_{c-1}\right) \text {. }
$$

A semigroup $S$ is called nilpotent of class $c$ (or $c$-nilpotent) if it satisfies $L_{c}$ for every $x, y \in S, z_{1}, z_{2}, \cdots z_{c-1} \in S^{1}$ where $S^{1}$ is the monoid obtained by adjoining the identity 1 to $S$. Note that we have slightly modified the definition of [14], allowing the variables $z$ to belong to $S^{1}$ (An equivalent version of Theorem 1 in [14] is: A semigroup can be embedded in a $c$-nilpotent group if and only if it is cancellative and $c$-nilpotent).

The next proposition provides some natural examples of nilpotent semigroups.

Proposition 2.1. Let $R$ be a commutative ring. Let $S$ be any semigroup of $n$ by $n(n>1)$ triangular matrices over $R$, each matrix in $S$ having equal entries in the main diagonal. Then $S$ is nilpotent of class $n-1$.

The proof depends on a simple property of the words $q_{c}$ serving in the definition of $L_{c}$. If $w=w\left(u_{1}, u_{2}, \cdots, u_{n}\right)$ is a word in the letters $u_{1}, u_{2}, \cdots, u_{n}$, we shall say that $w^{\prime}=w^{\prime}\left(u_{1}, u_{2}, \cdots, u_{n}\right)$ is extracted from $w$ if $w^{\prime}$ is obtained from $w$ by erasing letters in $w$.

Note that $w^{\prime}$ is extracted from $w$ if and only if $w^{\prime}$ is a monomial in the expansion of $w\left(1+u_{1}, 1+u_{2}, \cdots, 1+u_{n}\right)$ in the semigroup ring over the free monoid on $u_{1}, u_{2}, \cdots, u_{n}$. In view of this remark the following result is clear:

LeMma 2.2. For any word $w\left(x, y, z_{1}, z_{2}, \cdots, z_{c-1}\right)$ of length $l \leqq c$ that can be extracted from $q_{c}\left(x, y, z_{1}, z_{2}, \cdots, z_{c-1}\right)$ it is also possible to extract $w\left(y, x, z_{1}, z_{2}, \cdots, z_{c-1}\right)$.

Proof of Proposition 2.1. Since nilpotency is preserved by formation of subsemigroups, it is enough to show that the multiplicative semigroup of the ring $R_{n}$ of all $n$ by $n$ triangular matrices with equal diagonal entries is $(n-1)$-nilpotent. If $X \in R_{n}$ we can write $X=$ $x I+T(X)$ where $I$ is the identity matrix and where $T(X)$ is obtained from $X$ by replacing the entries $x$ in the diagonal of $X$ by zeros. Recalling that a product of $n$ upper triangular $n \times n$ matrices is 0 , a product of $m$ matrices $X_{i}=x_{i} I+T\left(X_{i}\right)$ in $R_{n}$ can be written

$$
X_{1} X_{2} \cdots X_{m}=\sum_{0 \leqq l \leqq m i n(m, n-1)} x_{1} x_{2} \cdots T\left(X_{i_{1}}\right) \cdots T\left(X_{i_{l}}\right) \cdots x_{m} .
$$

Our conventions concerning the summation are, that for a fixed $l$, we 
form all possible sums of products of $l$ matrices $T\left(X_{i_{k}}\right)$ multiplied by elements of $R$. For $l=0$ the corresponding term in the sum is $x_{1} x_{2} \cdots x_{m} I$. In particular

$q_{n-1}\left(X_{1}, X_{2}, \cdots, X_{n}\right)=\sum_{0 \leqq l \leqq n-1} q_{n-1}\left(x_{1}, x_{2}, \cdots, T\left(X_{i_{1}}\right), \cdots, T\left(X_{i_{l}}\right), \cdots, x_{n}\right)$.

By Lemma 2.2 and the commutativity of $R$ the sum on the right side of the preceding equality is symmetric in $X_{1}$ and $X_{2}$, which shows that $R_{n}$ is $(n-1)$-nilpotent.

The fact that groups of triangular matrices over a field, with equal nonzero entries in the main diagonal are nilpotent (see [8], Exercise II, p. 298) is a consequence of Proposition 2.1, together with Corollary 1 of [14].

Recall that a semigroup $S$ is regular if for every $a \in S$ there exists $x \in S$ such that $a x a=a$. A semilattice of groups is a semigroup which is a union of groups and has commuting idempotents. The structure of semilattices of groups modulo groups and group homomorphisms is described in [2] (Theorem 4.11, p. 128). Concerning undefined notions in the proof of the next proposition we adopt the terminology of [2].

Proposition 2.3. For a semigroup $S$ the following are equivalent

(1) $S$ is regular and c-nilpotent;

(2) $S$ is a semilattice of c-nilpotent groups.

Proof. $\quad(1) \Rightarrow(2)$. Assume $S$ is regular and $c$-nilpotent. Then each principal factor of $S$ is regular, 0-simple or simple ([2], Lemma 2.39) and $c$-nilpotent, since nilpotency is preserved by homomorphic image. By Theorem 2.54 of [2], each principal factor of $S$ is completely 0-simple or $S$ contains a copy of the bicyclic monoid $B$ presented by:

$$
<a, b ; a b=1>\text {. }
$$

But a monoid admitting the presentation (B) and $c$-nilpotent satisfies

$$
q_{c}(b a, b, 1, \cdots, 1)=q_{c}(b, b a, 1, \cdots, 1) \text {. }
$$

By induction on $c$ and using $a b=1$ we can write this equality in the form

$$
b^{2 c}=b^{2^{c}+1} a \text {. }
$$

It follows $a^{2 c} b^{2 c}=a^{2^{c}} b^{2 c+1} a$ or $b a=1$. Thus the only nilpotent monoid admitting the presentation (B) is the infinite cyclic group. This rules out the possibility $S$ containing a copy of the bicyclic monoid. On the other hand, a completely 0-simple semigroup which is $c$-nilpotent is a group with zero. To see this, assume that the Rees matrix semigroup $D=M^{0}(G ; I, A, P)$ is $c$-nilpotent. If the sandwich matrix 
$P$ has nonzero entries $p_{\lambda j}$ and $p_{\mu i}$, then replacing in $L_{c}, x$ by $(\alpha ; i, \lambda)$, $y$ by $(b ; j, \mu)(a, b \in G)$, and $z_{1}, z_{2}, \cdots, z_{c-1}$ by elements of $D$ such that both members of $L_{c}$ are not zero, we obtain $i=j$ and $\lambda=\mu$. The matrix $P$ having at least one nonzero entry in each row and column, it follows that $I$ and $\Lambda$ both have cardinality 1 , i.e., $D$ is a group with zero. From Theorem 4.6 of [2] we deduce that $S$ is a semilattice of groups.

$(2) \Rightarrow(1)$. A semilattice of groups is an inductive system $\left\{G_{\alpha}, \varphi_{\alpha \beta}, \Omega\right\}$ of groups $G_{\alpha}$ and group homomorphisms $\varphi_{\alpha \beta}: G_{\beta} \rightarrow G_{\alpha}$ indexed by elements of the semilattice $\Omega$. If $x \in G_{\alpha}, y \in G_{\beta}, z_{i} \in G_{\alpha_{i}}$,

$$
q_{c}\left(x, y, z_{1}, \cdots, z_{c-1}\right)=q_{c}\left[\varphi_{i \alpha}(x), \varphi_{\gamma \beta}(y), \varphi_{\gamma \alpha_{1}}\left(z_{1}\right), \cdots, \varphi_{\gamma \alpha_{c-1}}\left(z_{c-1}\right)\right]
$$

where $\gamma=\alpha \beta \alpha_{1} \cdots \alpha_{c-1}$. Thus, if the law $L_{c}$ holds for any group $G_{\alpha}, \alpha \in \Omega$, it holds for $S$. Consequently $S$ is $c$-nilpotent.

REMARKS. (1) The fact that all the groups occurring in a semilattice of groups $S$ are $c$-nilpotent (for a fixed $c$ ) is essential to ensure the nilpotency of $S$. For example, if $S$ is a chain of groups $G_{i}$ indexed by the integers ordered by $1>2>\cdots>i>i+1>\cdots$ with trivial connecting homomorphisms and with each $G_{i}$ of class strictly $i$, then $S$ is not nilpotent.

(2) From the proof of Proposition 2.3, we can deduce that in a nilpotent semigroup a given $\mathscr{J}$-class is either a nilpotent group or contains no idempotents.

3. Residual finiteness. A semigroup $S$ is residually finite if for every pair $a, b \in S, a \neq b$ there is a homomorphism $\varphi: S \rightarrow \varphi(S)$, with $\varphi(S)$ finite such that $\varphi(a) \neq \varphi(b)$. Free nilpotent semigroups are embeddable in free nilpotent groups. Thus they are residually finite since the latter are. By a result of Malcev, finitely generated semigroups of matrices over a field of characteristic 0 are residually finite [10]. It follows that the finitely generated semigroups in Proposition 2.1 are residually finite, provided $R$ is a field of characteristic 0 . If $R$ is an arbitrary ring we do not know if the result is still true. With the regularity condition we have the following consequence of Proposition 2.3.

COROLlARY 3.1. If a semigroup $S$ is finitely generated, nilpotent, and regular then $S$ is residually finite.

Proof. By Proposition 2.3, $S$ is a semilattice of nilpotent groups. Let $G_{e}$ be a maximal subgroup of $S$ with identity $e$. The mapping $\theta_{e}: S \rightarrow G_{e}^{0}$ defined by $\theta_{e}(x)=x e$ if $x e \in G_{e}$ and $\theta_{e}(x)=0$ if $x e \notin G_{e}$ is a 
homomorphism of $S$ onto $G_{e}^{0}$ (or $G_{e}$ if $G_{e}$ is the minimal ideal of $S$ ). Consequently, $G_{e}$ is finitely generated. Let $a \in G_{e}, b \in G_{f}$ be two distinct elements of $S$. If $f \neq e$, assume for example $f \geqq e(f \leqq e$ means $e f=f e=f)$. The homomorphism $\theta_{e}$ maps $S$ onto $G_{e}^{0}$ and $\theta_{e}(a)=a, \theta_{e}(b)=0$. If $\psi$ denotes the cannonical homomorphism from $G_{e}^{0}$ to $\{0,1\}$ we have $\psi \theta_{e}(a)=1$ and $\psi \theta_{e}(b)=0$. In case $f=e, \theta_{e}(a)=$ $a$ and $\theta_{e}(b)=b$. Since $G_{e}$ is finitely generated and nilpotent there is a homomorphism $\varphi$ of $G_{e}$ into a finite group such that $\varphi(a) \neq \varphi(b)$. Extending $\varphi$ to $G_{e}^{0}$ in a natural way, we see that $\psi \theta_{e}$ maps $S$ onto a finite group and separates $a$ and $b$.

Note that the structural semilattice of $S$ is finite, but we have not used this property in the proof. Indeed, we have the following weakened form of Corollary 3.1: If $S$ is nilpotent regular and has all its maximal subgroups finitely generated, then $S$ is residually finite.

4. Remarks on 2-nilpotent semigroups. A semigroup is called semisimple if each of its principal factor is simple or 0 -simple. The next proposition allows us to replace regularity in Proposition 3.1 by semisimplicity in the case of 2-nilpotent semigroups.

Proposition 4.1. If $S$ is a 0-simple 2-nilpotent semigroup, then $S$ is a group with zero.

Proof. We shall show that $S$ contains an idempotent. Then a similar argument as in the proof of Proposition 3.1, excluding the possibility of $S$ containing the bicyclic monoid and also the possibility of $S$ being properly completely 0 -simple, will give us the desired conclusion. Let $x, y \in S, x, y \neq 0$. Since $S$ is 0 -simple there exists $u, v \in S$ such that $x=u y v$ ([2], Lemma 2.28). Let us show that for every $z, t \in S$ there are elements $\alpha, \beta \in S$ such that $z=u \alpha t \beta v$. There are elements $r, r^{\prime}, s, s^{\prime} \in S$ such that $z=r x s$ and $y=r^{\prime} z s^{\prime}$. It follows $z=$ ruyvs $=r u r^{\prime} z s^{\prime} v s=\left(r u r^{\prime}\right)^{3} z\left(s^{\prime} v s\right)^{3}$. But in a 2-nilpotent semigroup $(a b)^{3}=b a^{3} b^{2}=a^{2} b^{3} a$. Thus

$$
z=u r^{\prime} r^{3}\left(u r^{\prime}\right)^{2} z\left(s^{\prime} v\right)^{2} s^{3} s^{\prime} v=u \alpha t \beta v
$$

for some $\alpha, \beta \in S$. Appling the property to $u$ and $v$ themselves, we can write $u=u \alpha_{1} v \beta_{1} v$ and $v=u \alpha_{2} u \beta_{2} v$. The first equality implies

$$
\begin{aligned}
u & =u \alpha_{1} v \beta_{1} v \alpha_{1} v \beta_{1} v=u v \alpha_{1} \beta_{1} \alpha_{1} v^{2} \beta_{1} v=u v \alpha_{1} \beta_{1} \alpha_{1} v u \alpha_{2} u \beta_{2} v \beta_{1} v \\
& =v u \alpha_{1} \beta_{1} \alpha_{1} u v \alpha_{2} u \beta_{2} v \beta_{1} v .
\end{aligned}
$$

(Pairs to which we have applied $L_{2}$ have been underlined.) Replacing $u$ in $v=u \alpha_{2} u \beta_{2} v$ by the expression $v \cdots v$ just obtained, we get $v=$ 
$v v^{\prime} v$ for some $v^{\prime} \in S$, establishing the existence of an idempotent $v v^{\prime}$ in $S$.

COROLlaRY 4.2. Semisimple 2-nilpotent semigroups are semilattices of 2-nilpotent groups and finitely generated semisimple 2nilpotent semigroups are residually finite.

Further attempts to remove the regularity condition from the statement of Corollary 3.1 encounter a major difficulty which lies essentially in the manipulation of nonregular $\mathscr{J}$-classes. To prove residual finiteness in the commutative case, a pleasant feature is that all the Green's relations coincide, which makes it possible to treat simultaneously regular and irregular $\mathscr{J}$-classes [7]. In spite of the fact that a regular $\mathscr{J}$-class of a nilpotent semigroup is a group, coincidences of the Green's relations are only accidental. In particular $\mathscr{D} \neq \mathscr{J}$ as shown in Example 4.5.

In order to simplify the computations in Example 4.5 we indicate canonical forms of words in the free 2-nilpotent semigroup with two generators $x, y$ (Proposition 4.4). By the relative length of a word $w$ in the letters $x, y, \cdots$, we mean the number of occurrences of distinct letters $x, y, \cdots$, in $w$, disregarding successive occurrences of the same letter. For example $x^{5} y^{3} x^{2} y x^{6} y$ has relative length 6 .

LEMMA 4.3. In the free 2-nilpotent semigroup with 2 generators, any word can be written as a word of relative length at most 5.

Proof. It is sufficient to show that the relative length of $w=$ $x y^{m} x^{n} y^{p} x^{q} y$ where $m, n, p, q \geqq 1$ can be reduced. This is done by induction on the total degree $d_{x}(w)$ of $w$ in the letter $x$, using the 2nilpotent law. Note that the induction process shows that any word starting with $x$ can be reduced to a word of relative length at most 5 starting with $x$. If a word of length 5 starts with $y$ and cannot be transformed into a word starting with $x$, then it can be reduced to length at most 4 , as follows:

$$
\begin{aligned}
y^{\alpha} x^{\beta} y^{\gamma} x^{\delta} y^{\varepsilon} & =y^{\alpha} x x^{\beta-1} y^{\gamma} x^{\delta} y^{\varepsilon}=y^{\alpha-\delta \varepsilon} x y^{\delta \varepsilon} x^{\beta-1} y^{\gamma+\varepsilon} x^{\delta} \\
& =y^{\alpha-\delta \varepsilon-(\beta-1)(\gamma+\varepsilon)} x y^{\delta \varepsilon+\beta(\delta+\varepsilon)} x^{\beta-1+\delta}
\end{aligned}
$$

REMARK. The property of the lemma does not hold for 2-nilpotent semigroups with more than 2 generators. In 3 generators $x, y, z$ consider e.g. $x y z x^{2} y^{2} z^{2} \cdots x^{n} y^{n} z^{n}$.

Proposition 4.4. In the free 2-nilpotent semigroup with 2 generators $x, y$, any word $w$ has a unique expression of the form $x^{\alpha} y^{\gamma} x^{\alpha^{\prime}}$ or 
$x^{\alpha} y^{\beta} x y^{\beta^{\prime}} x^{\alpha^{\prime}}$ where [resp. $\alpha^{\prime}$ ] is the largest possible power of $x$ with which $w$ starts [resp. ends] and [resp. $\beta^{\prime}$ ] is the smallest possible power of $y$ in the first [resp. last] occurrences of $y^{\prime} s$ in $w$.

Proof. Among all expressions of $w$ as a word of relative length 5 , let $x^{\alpha} y^{m} x^{n} y^{p} x^{q}$ be an expression in which the power $\alpha$ of the first $x$ is maximal $(\alpha \geqq 0)$. If $n \geqq 2, m \geqq 1, p \geqq 1$,

$$
\begin{aligned}
w=x^{\alpha} y^{m} x^{n} y^{p} x^{q} & =x^{\alpha} y^{m-1}(y x) x^{n-2}(x y) y^{p-1} x^{q} \\
& =x^{\alpha} y^{m-1}\left(x y x^{n-2} y x y^{p-1} x^{q}\right) .
\end{aligned}
$$

Thus $w=x^{\alpha} y^{m-1} w_{1}$ with $w_{1}$ of relative length at most 5. Pursuing the process $w$ can be written as $x^{\alpha} y^{\beta} w_{0}$ with $w_{0}$ of length at most 5 , starting with $x$ and $\beta$ minimal. By the minimality of $\beta, w_{0}$ cannot contain more than one occurrence of $x y$, thus $w_{0}=x y^{\beta^{\prime}} x^{\alpha^{\prime}}$ and $w=$ $x^{\alpha} y^{\beta} x y^{\beta^{\prime}} x^{\alpha^{\prime}}$. From the obtained form for $w, \alpha^{\prime}$ is also maximal and $\beta^{\prime}$ minimal.

It is worth noting that Lemma 4.3 and Proposition 4.4 provide an effective algorithm for solving the word problem in the free 2-nilpotent semigroup with two generators.

ExAmPLE 4.5. Let $S$ be the 2-nilpotent semigroup with zero, presented by

$$
<x, y ; x=y x y, x^{2}=0>\text {. }
$$

The presentation of $S$ together with the 2-nilpotency law imply $x y^{m} x=0$ for every $m \geqq 0$. By Proposition 4.5, elements of $S$ have the canonical forms $y^{p}, y^{m} x, x y^{n}(p>0, m, n \geqq 0)$. The reader may check that all the Green's relations, except $\mathscr{J}$ coincide with the equality. All the $\mathscr{J}$-classes are singleton except one of them $J=$ $\left\{x, y^{m} x, x y^{n}, m, n>0\right\}$. Thus $\mathscr{D} \neq \mathscr{J}$. To show that $S$ is residually finite, there is no difficulty in separating elements lying in distinct $\mathcal{F}$-classes with the use of Rees congruences. To separate distinct elements in $J$, it is enough to add a relation of the form $y=y^{k}$ to the presentation of $S$.

\section{REFERENCES}

1. W. H. Carlisle, Residual finiteness of finitely generated commutative semigroups, Pacific J. Math., 36 (1971), 99-101.

2. A. H. Clifford and G. B. Preston, The algebraic theory of semigroups, Vol. 1, Math. Surveys No. 7, Amer. Math. Soc., Providence, R. I., 1961.

3. T. Evans, Some connections between residual finiteness, finite embeddability and the word problem, J. London Math. Soc., (2), 1 (1969), 399-403.

4. — Finitely presented loops, lattices, etc. are hopfian, J. London Math. Soc., 44 (1969), 551-552. 
5. — Residually finite semigroups of endomorphisms, J. London Math. Soc., 2 (1970), 719-721.

6. K. Hirsch, On infinite solvable groups III, Proc. London Math. Soc., (2), 49 (1946), 184-194.

7. G. Lallement, On a theorem of Malcev, Proc. Amer. Math. Soc., 30 (1971), 49-54.

8. W. Magnus, A. Karrass and D. Solitar, Combinatorial Group Theory, Wiley, New York, 1966.

9. W. Magnus, Residually finite groups, Bull. Amer. Math. Soc., 75 (1969), 305-316.

10. A. I. Malcev, On isomorphic matrix representations of infinite groups, Mat. Sbornik 8 (50), (1940), 405-422 (in Russian).

11. - Nilpotent semigroups, Uch. Zap. Ivanov, Gos. Ped. Inst. 4 (1953), 107-111 (in Russian).

12. On homomorphisms onto finite groups, Uch. Zap. Ivanov. Gos. Ped. Inst. 18 (1958), 49-60 (in Russian).

13. J. C. C. McKinsey, The decision problem for some classes and sentences without quantifiers, J. Symbolic Logic, 8 (50), (1940), 405-422.

14. B. H. Neumann and Tekla Taylor, Subsemigroups of nilpotent groups, Proc. Roy. Soc., Ser. A, 274 (1963), 1-4.

15. E. L. Post, Recursive unsolvability of a problem of Thue, J. Symbolic Logic, 12 (1947), 1-11.

Received April 22, 1971 and in revised form June 7, 1972.

The Pennsyluania State University 


\section{PACIFIC JOURNAL OF MATHEMATICS}

\section{EDITORS}

\section{H. SAMELSON}

Stanford University

Stanford, California 94305

\section{R. HOBBY}

University of Washington

Seattle, Washington 98105

\section{J. DugundjI}

Department of Mathematics University of Southern California Los Angeles, California 90007

RICHARD ARENS

University of California

Los Angeles, California 90024

\section{ASSOCIATE EDITORS}
E. F. BECKENBACH
B. H. NeumanN
F. WOLF
K. YOSHIDA

\section{SUPPORTING INSTITUTIONS}

\author{
UNIVERSITY OF BRITISH COLUMBIA \\ CALIFORNIA INSTITUTE OF TECHNOLOGY \\ UNIVERSITY OF CALIFORNIA \\ MONTANA STATE UNIVERSITY \\ UNIVERSITY OF NEVADA \\ NEW MEXICO STATE UNIVERSITY \\ OREGON STATE UNIVERSITY \\ UNIVERSITY OF OREGON \\ OSAKA UNIVERSITY
}

\author{
UNIVERSITY OF SOUTHERN CALIFORNIA \\ STANFORD UNIVERSITY \\ UNIVERSITY OF TOKYO \\ UNIVERSITY OF UTAH \\ WASHINGTON STATE UNIVERSITY \\ UNIVERSITY OF WASHINGTON \\ AMERICAN MATHEMATICAL SOCIETY \\ NAVAL WEAPONS CENTER
}

The Supporting Institutions listed above contribute to the cost of publication of this Journal, but they are not owners or publishers and have no responsibility for its content or policies.

Mathematical papers intended for publication in the Pacific Journal of Mathematics should be in typed form or offset-reproduced, (not dittoed), double spaced with large margins. Underline Greek letters in red, German in green, and script in blue. The first paragraph or two must be capable of being used separately as a synopsis of the entire paper. The editorial "we" must not be used in the synopsis, and items of the bibliography should not be cited there unless absolutely necessary, in which case they must be identified by author and Journal, rather than by item number. Manuscripts, in duplicate if possible, may be sent to any one of the four editors. Please classify according to the scheme of Math. Rev. Index to Vol. 39. All other communications to the editors should be addressed to the managing editor, Richard Arens, University of California, Los Angeles, California, 90024.

50 reprints are provided free for each article; additional copies may be obtained at cost in multiples of 50 .

The Pacific Journal of Mathematics is published monthly. Effective with Volume 16 the price per volume (3 numbers) is $\$ 8.00$; single issues, $\$ 3.00$. Special price for current issues to individual faculty members of supporting institutions and to individual members of the American Mathematical Society: $\$ 4.00$ per volume; single issues $\$ 1.50$. Back numbers are available.

Subscriptions, orders for back numbers, and changes of address should be sent to Pacific Journal of Mathematics, 103 Highland Boulevard, Berkeley, California, 94708.

PUBLISHED BY PACIFIC JOURNAL OF MATHEMATICS, A NON-PROFIT CORPORATION

Printed at Kokusai Bunken Insatsusha (International Academic Printing Co., Ltd.), 270, 3-chome Totsuka-cho, Shinjuku-ku, Tokyo 160, Japan. 


\section{Pacific Journal of Mathematics}

\section{Vol. 42, No. $3 \quad$ March, 1972}

Catherine Bandle, Extensions of an inequality by Pólya and Schiffer for vibrating membranes ................................ 543

S. J. Bernau, Topologies on structure spaces of lattice groups.......... 557

Woodrow Wilson Bledsoe and Charles Edward Wilks, On Borel product measures .......................................

Eggert Briem and Murali Rao, Normpreserving extensions in subspaces of

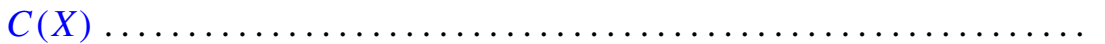

Alan Seymour Cover, Generalized continuation.................. 589

Larry Jean Cummings, Transformations of symmetric tensors .......... 603

Peter Michael Curran, Cohomology of finitely presented groups .......... 615

James B. Derr and N. P. Mukherjee, Generalized quasicenter and

hyperquasicenter of a finite group ...................... 621

Erik Maurice Ellentuck, Universal cosimple isols .................. 629

Benny Dan Evans, Boundary respecting maps of 3-mainfolds .......... 639

David F. Fraser, A probabilistic method for the rate of convergence to the

Dirichlet problem .................................. 657

Raymond Taylor Hoobler, Cohomology in the finite topology and Brauer

groups ..................................... 667

Louis Roberts Hunt, Locally holomorphic sets and the Levi form ........ 681

B. T. Y. Kwee, On absolute de la Vallée Poussin summability............ 689

Gérard Lallement, On nilpotency and residual finiteness in semigroups .... 693

George Edward Lang, Evaluation subgroups of factor spaces........... 701

Andy R. Magid, A separably closed ring with nonzero torsion pic ....... 711

Billy E. Rhoades, Commutants of some Hausdorff matrices ............. 715

Maxwell Alexander Rosenlicht, Canonical forms for local derivations . . . . 721

Cedric Felix Schubert, On a conjecture of L. B. Page ................ 733

Reinhard Schultz, Composition constructions on diffeomorphisms of $S^{p} \times S^{q}$

J. P. Singhal and H. M. (Hari Mohan) Srivastava, A class of bilateral generating functions for certain classical polynomials ....

Richard Alan Slocum, Using brick partitionings to establish conditions which insure that a Peano continuum is a 2-cell, a 2-sphere or an annulus...

James F. Smith, The p-classes of an $H^{*}$-algebra ...

Jack Williamson, Meromorphic functions with negative zeros and positive

poles and a theorem of Teichmuller ................. 